\title{
Long-term effects of pallidal deep brain stimulation in tardive dystonia: a follow-up of 5-14 years
}

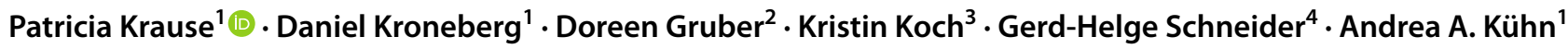

Received: 4 October 2021 / Revised: 5 January 2022 / Accepted: 6 January 2022 / Published online: 27 January 2022

(c) The Author(s) 2022

\begin{abstract}
Introduction Pallidal DBS is an established treatment for severe isolated dystonia. However, its use in disabling and treatment-refractory tardive syndromes (TS) including tardive dyskinesia and tardive dystonia (TD) is less well investigated and long-term data remain sparse. This observational study evaluates long-term effects of deep brain stimulation (DBS) of the globus pallidus internus (GPi) in patients with medically refractory TS.

Methods We retrospectively analyzed a cohort of seven TD patients with bilateral GPi-DBS. Involuntary movements, dystonia and disability were rated at long-term follow-up (LT-FU) after a mean of 122 \pm 33.2 SD months (range 63-171 months) and compared to baseline (BL), short-term (ST-FU; mean $6 \pm 2.0$ SD months) and 4-year follow-up (4y-FU; mean $45 \pm 12.3$ SD months) using the Abnormal Involuntary Movement Scale (AIMS) and the Burke-Fahn-Marsden Dystonia Rating Scale (BFMDRS), respectively. Quality of life and mood were evaluated using the SF36 and Beck Depression Index (BDI) questionnaires, respectively.

Results At LT-FU patients had improved by $73 \% \pm 14.2$ SD in involuntary movements and $90 \% \pm 1.0$ SD in dystonia. Mood had improved significantly whereas quality of life remained unchanged compared to baseline. No serious long-lasting stimulation-related adverse events (AEs) were observed. Three patients of this cohort presented without active stimulation and ongoing symptom relief at long-term follow-up after 3-10 years of continuous DBS.

Conclusion Pallidal DBS is a safe and effective long-term TD treatment. Even more interesting, three of our patients could stop stimulation after several years of DBS without serious relapse. Larger studies need to explore the phenomenon of ongoing symptom relief after DBS cessation.
\end{abstract}

Keywords Dystonia $\cdot$ Tardive $\cdot$ Pallidal DBS $\cdot$ Long-term effects $\cdot$ DBS and quality of life

Andrea A. Kühn

andrea.kuehn@charite.de

1 Movement Disorder and Neuromodulation Unit, Department of Neurology, Charité, University Medicine Berlin, Campus Mitte, Charitéplatz 1, 10117 Berlin, Germany

2 Department of Neurology and Stereotactic Surgery, University Medicine of Magdeburg, Magdeburg, Germany

3 Department of Psychiatry and Psychotherapy, Charité, University Medicine Berlin, Campus Mitte, Berlin, Germany

4 Department of Neurosurgery, Charité, University Medicine Berlin, University Medicine Berlin, Campus Mitte, Berlin, Germany

\section{Introduction}

Tardive syndromes (TS) encompass a broad spectrum of abnormal involuntary movements (AIMs) of the tongue, jaw, trunk and/or extremities emerging after at least 3 months of exposure to dopamine receptor blocking agents, but also after treatment with certain antiemetics and antidepressants [1]. Clinical presentation of TS varies widely between, e.g. dyskinetic, dystonic, stereotypic, tremulous, choreiform and athetoid movements [1]. Up to $21 \%$ of the patients treated with dopamine receptor blocking agents (DRBA) are estimated to develop tardive symptoms [1]. TS is often associated with stigmatization and incapacity causing socioemotional distress leading to increased mortality and morbidity [2]. Treatment of TS is challenging and often disappointing. First and foremost, causative drugs ought to be avoided. Other medical therapeutic regimen include 
dosage reduction, substitution of atypical neuroleptics and the probatory use of tetrabenazine, anticholinergics, botulinum toxin, amantadine, benzodiazepines, propranolol or antioxidants [1]. A recent review reported that more than $50 \%$ of TS cases were irreversible after withdrawal from the responsible neuroleptics [3]. The remission rate of TS is yet unclear ranging between 2 and $12 \%$ after up to 4 years of discontinuation or reduction of mostly DRBA [1]. Deep brain stimulation (DBS) of the globus pallidus internus (GPi) is an effective treatment for medically refractory dystonia and has progressively evolved into a widely available therapeutic strategy in dystonia as it reduces not only motor impairment but also disability [4]. However, compared to isolated dystonia [5], its use in disabling and treatment refractory TS including tardive dyskinesia and tardive dystonia (TD) is less well investigated. According to the most recent reviews, 24 single case reports and 6 rather small open-label case series reported GPi-DBS to be a safe and promising treatment option, with improvements between 30 and $90 \%$ on disease-specific scales after up to 7 years [6-8]. Among the three controlled studies [9-11], the only randomized, sham-controlled trial using a delayed-start design of pallidal neurostimulation in TD did not reach a significant difference between sham and active stimulation in the blinded, controlled phase [10]. At 6-month open follow-up, however, the study cohort showed a mean $40 \%$ improvement of dystonia along with improvements in quality of life (QoL) [10]. Similarly, the French STARDY group reported 50\% motor improvement in 10 patients after 6 months of GPi-DBS [9]. The longest follow-up, so far, has been reported in 14 TD patients after 6-11 years of GPi-DBS with an overall 63 and 58\% motor improvement measured by the AIMS and extrapyramidal symptom rating scale, respectively [11]. Long-term outcome as well as information on QoL, mood and side effects of this potentially life-long therapy are of special clinical interest.

\section{Methods}

\section{Patients}

Seven TD-patients (six women) with pallidal DBS operated between 2001 and 2008 at the University Hospital Charité, Berlin, Germany, were available for long-term followup after 5 to 14 years of stimulation. Patients had a mean disease duration of $65.2 \pm 48.4$ months (mean \pm standard deviation; range 12-132 months) and a mean age at surgery of $57.6 \pm 17.4$ years (range $30-75$ years). TD was either attributed to prior antipsychotic or antiemetic treatment. None of the patients responded to various medical treatment attempts (see Suppl. Table). Long-term follow-up presented here (LT-FU; mean $121.7 \pm 33.2$ months; range
63-171 months) includes assessment of motor impairment, mood, QoL, cognition, stimulation parameters, DBS side effects, medication (see Table 1 and Suppl. Table 1). These data were compared retrospectively to data from baseline (BL; preoperative), short-term follow-up (ST-FU; mean $6 \pm 1.9$ months; range 4-9 months) and 4-year follow-up (4y-FU; mean $44.9 \pm 12.3$ months; range $26-65$ months) that were all video documented $[10,12]$. The study was approved by the local ethics committee. All patients gave their written informed consent.

\section{Clinical assessments}

LT-FU assessment of motor symptoms was performed in a non-blinded fashion employing the Abnormal Involuntary Movement Scale (AIMS) for tardive dyskinesia and the Burke-Fahn-Marsden Dystonia Rating Scale (BFMDRS) for dystonia severity and disability [13, 14]. Individual effects of stimulation on health-related QoL were assessed using the SF36 [15]. Occurrence of depressive symptoms was assessed using the Beck Depression Index (BDI) or the Hamilton depression scale (HAM-D) [16, 17]. Cognition was rated by use of Mini-Mental State Exam (MMSE) or Mattis dementia rating scale [18, 19]. All scores were compared to available archival BL and 4y-FU data. All reported device-related side effects and adverse events (AEs) were collected retrospectively from archival records. Patients were additionally asked about chronic side effects or AEs at LT-FU.

\section{Statistical analysis}

Motor function, disability and QoL data were compared between BL and the different FU time points using Friedman test and post hoc Wilcoxon test. A Spearman's correlation was done to investigate possible correlations between motor outcome and demographic factors such as age at onset, disease severity, changes in QoL and mood (SF-36 and BDI). All data are given as mean $\pm \mathrm{SD}$ if not mentioned otherwise. A $P$ value $<0.05$ was considered to be significant.

\section{Results}

Mean motor scores were significantly improved at LT-FU compared to preoperative baseline for AIMS and for BFMDRS motor scores leading to a mean improvement of $73 \pm 14.2 \%$ and $90 \pm 1.0 \%$, respectively. BFMDRS disability score improved by $79 \pm 1.1 \%$ at LT-FU. All patients presented with mild dystonic features before surgery that were predominantly affecting head/neck and upper extremities. Subgroup analysis of the BFMDR motor score revealed a predominant antidystonic effect on the subitems 


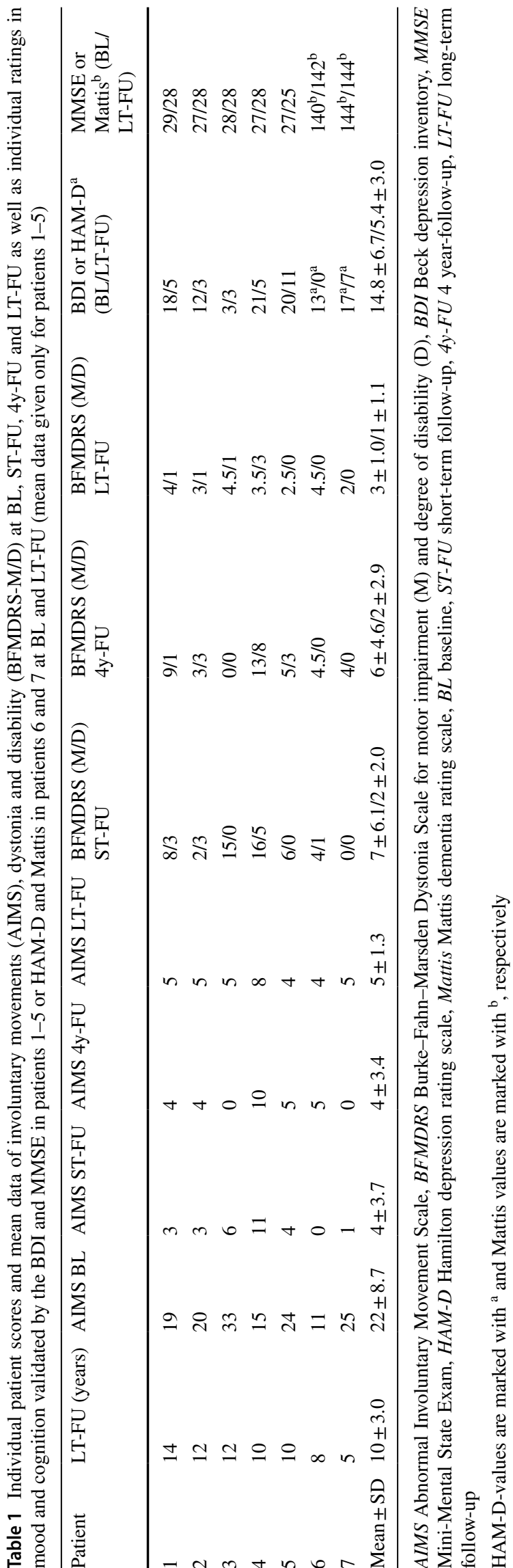

mouth $(-4.8$ pts. \pm 1.2 at $4 \mathrm{y}-\mathrm{FU} ;-5.2 \mathrm{pts} \pm 1.5$ at LT-FU; $\mathrm{p}=0.0002$ and 0.0003 , respectively) and neck $(-5.1$ pts. \pm 2.5 at $4 \mathrm{y}-\mathrm{FU} ;-5.7 \mathrm{pts} \pm 2.4$ at LT-FU; $\mathrm{p}=0.002$ and 0.0008 , respectively). The DBS-induced improvement was sustained and consistent over the follow-up period (Fig. 1A-C and Table 1). Importantly, at LT-FU, only patients 1-4 presented with activated pallidal stimulation, whereas patients 5-7 had discontinued stimulation without significant deterioration of TD for $29.3 \pm 22.1$ months (range 6-59 months; Fig. 1 D). QoL ratings using the SF36 revealed no significant change at LT-FU $(59.5 \pm 28.27$ points $)$ in comparison to BL ( $53 \pm 30.8$ points). Sub-analysis of the SF36 physical component score (PCS) versus the SF36 mental component score (MCS) showed a tendency towards higher improvements in the MCS with $53 \pm 26$ points (range $0-100$ ) at baseline and $62.6 \pm 21.9$ points (range $0-100$ ) at LT-FU versus $55 \pm 36$ points (range $0-100$ ) at baseline and $56.5 \pm 34$ points (range 0-100) in the PCS. However, this difference was not statistically significant. Mean BDI depression score in patients $1-5$ were significantly decreased at LT-FU and showed stable reduction in HAM-D in patient $6+7$ (see Table 1). Cognition remained stable over time (as assessed with MMSE in patients 1-5 and Mattis score in patient $6+7$; see Table 1). There was no correlation between motor or non-motor improvement with DBS and age of onset or disease duration (data not shown). The main reason for surgical intervention after electrode implantation was IPG replacement after battery exemption with on average 1.6 $\pm 0.4 \mathrm{IPG}$ replacements (range 1-3) in patients $1-5$ over a period of 122 months (range 117-171) and a mean replacement interval of $47.3 \pm 7.1$ months (range 36-54). Patients 6 and 7 did not reach battery exemption. Patient 6 had the IPG explanted 8 years after implantation due to symptom remission. Four out of seven patients had switched to rechargeable stimulation devices at LT-FU. Since initial implantation, two adverse events (AE) were device related (tension along lead wires) with one being classified as serious adverse events (SAE) requiring surgical intervention due to intolerable pain alongside the lead wires. Patient 4 reported on transient stimulation-related dysarthria and gait disturbances remitting after stimulation parameter adjustments. No long-term side effects such as bradykinesia or gait disturbances were observed in our cohort.

\section{Discussion}

Here we present a retrospective long-term observation of more than 5 years of a patient cohort with severe tardive dystonia and therapeutic bilateral pallidal neurostimulation. The patients in our TD cohort presented with $70 \%$ reduction of involuntary movements and about $90 \%$ reduction of dystonic symptoms after 5-14 years of pallidal 
A

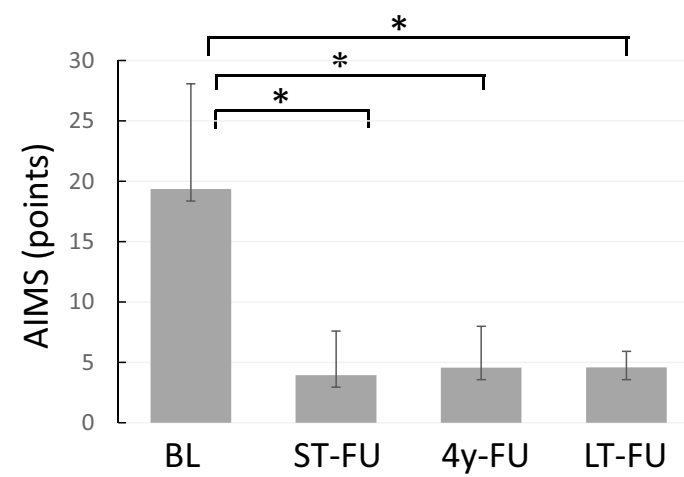

C

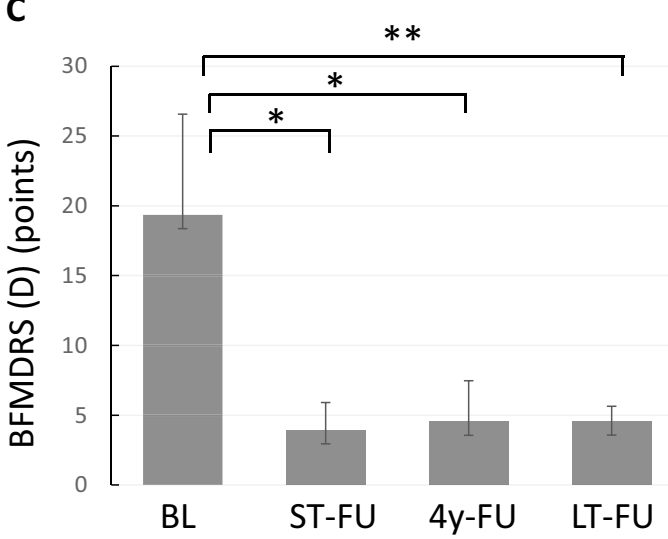

Fig. 1 A Mean absolute AIMS score at baseline (BL), short-term FU (ST-FU), mean 4-year follow-up (4y-FU) and mean long-term followup (LT-FU). B, C Mean absolute BFMDR motor and disability score at BL, ST-FU, 4y-FU and LT-FU. (D) Individual AIMS and BFMDR

DBS. This excellent outcome is in line with other reports on pallidal DBS in TD with especially good suppression of phasic and/or tremulous dystonic movements [20,21] whereas larger studies showed smaller DBS effects [10, 11]. While our patient cohort showed predominant phasic or tremolous dystonic movements, the higher variability of clinical responses across studies may be related to clinical subtypes of tardive syndromes. All our patients presented with mild dystonic features at baseline predominantly affecting head, neck, and upper extremities. While several studies reported on equal responses of affected dystonic body regions $[11,22]$, others found orobuccal-lingual dystonia to be less responsive than cervical dystonia [23]. Interestingly, our patients demonstrated similar motor effects for oro-buccal-lingual symptoms, axial and limb dystonia after $60-80$ months of pallidal stimulation [12] with a predominant amelioration in the subitems 'mouth' and 'neck' in the long-term follow-up subgroup analysis. This goes in line with other observations presenting gradual improvements of particular body regions over time requiring more and longer programming sessions to achieve meaningful improvement [24].
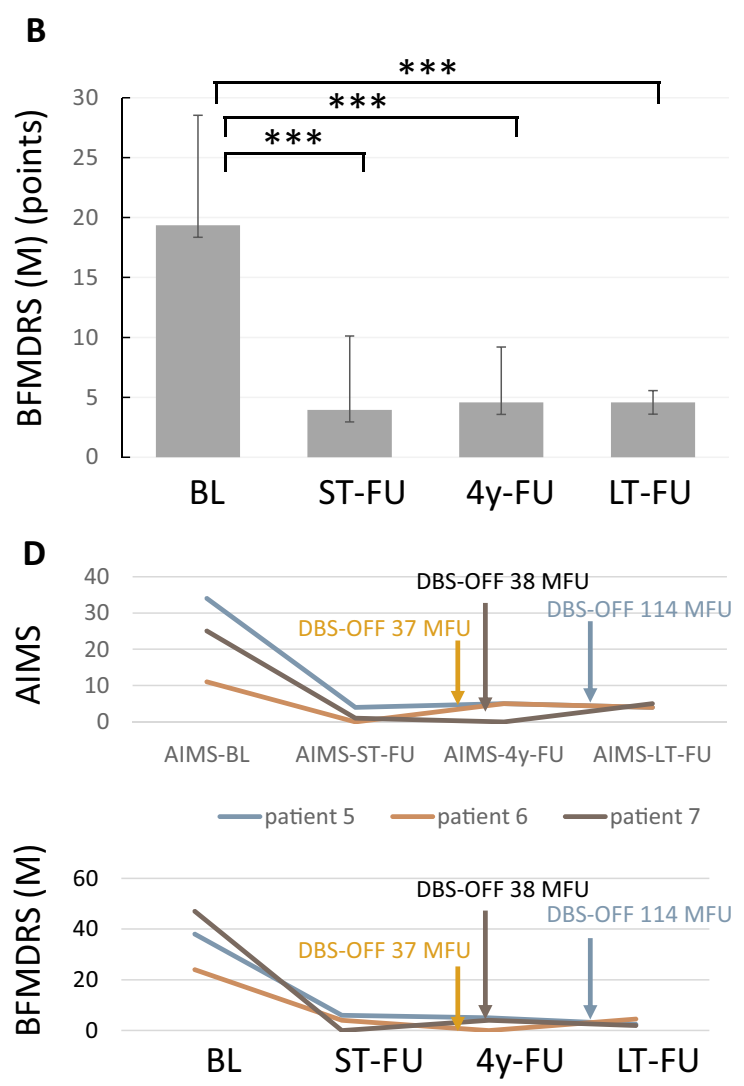

motor scores for patients 5-7 in whom DBS was switched OFF after more than 3 years of continuous pallidal stimulation. Time points for DBS-OFF at individual follow-up months (MFU) are marked with an arrow. * $p<0.05 ; * * p<0.005 ; * * * p<0.005$

All our patients suffered from dystonic symptoms in multiple body parts (see Suppl. Table 2). Patients 5-7 that could stop DBS after several years of continuous treatment initially presented with akathisia and/or high-frequency tremor additionally to their dystonic symptoms (see suppl. video); however, the general clinical pattern of dystonia did not differ in those patients. Nevertheless, patients 5-7 showed persistent remission of symptoms when DBS was stopped after continuous treatment for 10 (patient 5) and 3 (patients 6 and 7) years, respectively. Importantly, those patients had a disease duration of 2, 4, 5 years before surgery and showed a clinically relevant reoccurrence of involuntary movements during OFF testing at short-term follow-up (ST-FU visit). Consequently, it is rather unlikely that the remission reflects the natural course of the disease. While maintenance of benefits after DBS interruption was reported episodically in isolated dystonia [25, 26], several groups have rather reported on reappearance and even rebound of TD after discontinuation of stimulation up to now $[11,27,28]$. In line with this, battery depletion in patient 3 immediately resulted in recurrence of involuntary movements after 11 years of GPi-DBS. 
Only little is known about spontaneous remission rate in TD. Several groups reported on symptom reduction rather than remission in less than $13 \%$ of the patients within 2 to 4 years of discontinuation of the triggering pharmaceutical [29-31]. Prior medication, underlying disease, age at onset, age at surgery or stimulation parameters did not differ between stimulated and non-stimulated patients at LT-FU. The OFF-subgroup had already suffered from TD for 2-7 years before surgery and experienced another 3-10 years of continuous DBS before they were switched off permanently. Consequently, it is rather unlikely that the remission reflects the natural course of the disease. Interestingly, the initial clinical presentation in patients 5-7 was dominated by akathisia and/or highly frequent tremor additionally to dystonic features and variable oromandibular symptoms (see suppl. video). In contrast, generalized dystonic features dominated the clinical picture in patients 1-4. As we cannot but speculate if DBS may induce long-term neuroplastic changes in specific subtypes of TD, this needs to be explored in larger cohorts and by means of complementary electrophysiological and imagebased pre- versus post-interventional approaches in clinically well-characterized patients.

Gruber et al. found a significant $46 \%$ improvement limited to the physical health SF36 subscore and a trend towards improvements in all other subscores at 4-yr FU in the initial cohort, which includes patients $1-5$ of the present cohort [12]. Our patients showed a tendency towards higher improvements in the mental component subscore of the SF36 at long-term follow-up. Overall SF36 scores at LT-FU, however, showed no significant change compared to baseline. One has to keep in mind that the mean age of the total cohort was meanwhile $\sim 70$ years, and patients suffered from several additional comorbidities with potential impact on QoL. Interestingly, we observed a sustained improvement of depressive symptoms in our patients at LT-FU and no psychiatric adverse events. Psychiatric preconditions traditionally represent a relative contraindication for neurostimulation in DBS studies. No psychiatric adverse events occurred in our patients, which supports the conclusion of a recent review of 117 TD patients with DBS [6] and confirms that pallidal DBS is safe and effective in TD. Nevertheless, we have to consider that our study includes only a small sample size, non-blinded evaluation of stimulation benefits and probable interrater variability due to the retrospective character of the study. However, our study provides a comprehensive assessment of motor features, disability, QoL and mood in a long-term follow-up on an older TD population. Importantly, psychiatric comorbidity was not aggravated by DBS, supporting the recommendation of pallidal DBS in pharmacologically refractory severe TD with psychiatric stable condition. The most interesting observation is the persisting symptom remission in three out of seven patients after 3-10 years of chronic pallidal stimulation.

Supplementary Information The online version contains supplementary material available at https://doi.org/10.1007/s00415-022-10965-8.

Acknowledgements The study was supported by a grant from the German Research Foundation (DFG SFB TRR 295, Project-ID 424778381). PK was supported by the Charité Lydia Rabinowitsch grant; DK is supported by the clinician scientist program at the Charite, BIH. This study has been approved by the local ethics committee and has, therefore, been performed in accordance with the ethical standards laid down in the 1964 Declaration of Helsinki and its later amendments.

Author contributions (1) The conception and design of the study, or acquisition of data, or analysis and interpretation of data; (2) drafting the article or revising it critically for important intellectual content; (3) final approval of the version to be submitted. PK: 1, 2, and 3. DK: 2 . DG: 1 and 2. KK: 1. GHS: 1. AAK: 1, 2, and 3.

Funding Open Access funding enabled and organized by Projekt DEAL.

\section{Declarations}

Conflicts of interest AAK received speaker's honoraria and consultancies from Medtronic and Boston Scientific. P.K. received speaker's honoraria from Medtronic. D.K. is a participant in the BIH-Charité Clinician Scientist Program funded by the Charité-Universitätsmedizin Berlin and the Berlin Institute of Health. D.G. has received honoraria for speaking from Medtronic, outside the submitted work. K.K.: none G.H.S. received speaker's honoraria from Medtronic, Boston Scientific, and Abbott.

Ethical approval Our study has been approved by the appropriate ethics committee and have therefore been performed in accordance with the ethical standards laid down in the 1964 Declaration of Helsinki and its later amendments. All participants gave their informed consent prior to their inclusion in the study.

Open Access This article is licensed under a Creative Commons Attribution 4.0 International License, which permits use, sharing, adaptation, distribution and reproduction in any medium or format, as long as you give appropriate credit to the original author(s) and the source, provide a link to the Creative Commons licence, and indicate if changes were made. The images or other third party material in this article are included in the article's Creative Commons licence, unless indicated otherwise in a credit line to the material. If material is not included in the article's Creative Commons licence and your intended use is not permitted by statutory regulation or exceeds the permitted use, you will need to obtain permission directly from the copyright holder. To view a copy of this licence, visit http://creativecommons.org/licenses/by/4.0/.

\section{References:}

1. Aquino CC, Lang AE (2014) Tardive dyskinesia syndromes: current concepts. Parkinsonism Relat Disord 20(Suppl 1):S113-S117 
2. Youssef HA, Waddington JL (1987) Morbidity and Mortality in tardive-dyskinesia-associations in chronic-schizophrenia. Acta Psychiatr Scand 75(1):74-77

3. O'Brien A (2016) Comparing the risk of tardive dyskinesia in older adults with first-generation and second-generation antipsychotics: a systematic review and meta-analysis. Int J Geriatr Psychiatry 31(7):683-693

4. Vidailhet $\mathrm{M}$ et al (2005) Bilateral deep-brain stimulation of the globus pallidus in primary generalized dystonia. N Engl J Med 352(5):459-467

5. Moro E et al (2017) Efficacy of pallidal stimulation in isolated dystonia: a systematic review and meta-analysis. Eur J Neurol 24(4):552-560

6. Macerollo A, Deuschl G (2018) Deep brain stimulation for tardive syndromes: Systematic review and meta-analysis. J Neurol Sci 389:55-60

7. Mentzel CL et al (2012) Efficacy and safety of deep brain stimulation in patients with medication-induced tardive dyskinesia and/or dystonia: a systematic review. J Clin Psychiatry 73(11):1434-1438

8. Morigaki $\mathrm{R}$ et al (2016) Therapeutic perspective on tardive syndrome with special reference to deep brain stimulation. Front Psychiatry 7:207

9. Damier P et al (2007) Bilateral deep brain stimulation of the globus pallidus to treat tardive dyskinesia. Arch Gen Psychiatry 64(2):170-176

10. Gruber D et al (2018) Neurostimulation in tardive dystonia/dyskinesia: A delayed start, sham stimulation-controlled randomized trial. Brain Stimul 11(6):1368-1377

11. Pouclet-Courtemanche $\mathrm{H}$ et al (2016) Long-term efficacy and tolerability of bilateral pallidal stimulation to treat tardive dyskinesia. Neurology 86(7):651-659

12. Gruber D et al (2009) Long-term effects of pallidal deep brain stimulation in tardive dystonia. Neurology 73(1):53-58

13. Lane RD et al (1985) Assessment of tardive dyskinesia using the Abnormal Involuntary Movement Scale. J Nerv Ment Dis 173(6):353-357

14. Burke RE et al (1985) Validity and reliability of a rating scale for the primary torsion dystonias. Neurology 35(1):73-77

15 Ware JE Jr, Sherbourne CD (1992) The MOS 36-item short-form health survey (SF-36). I. Conceptual framework and item selection. Med Care 30(6):473-483

16. Beck AT et al (1961) An inventory for measuring depression. Arch Gen Psychiatry 4:561-571
17. Hamilton M (1960) A rating scale for depression. J Neurol Neurosurg Psychiatry 23:56-62

18 Folstein MF, Folstein SE, McHugh PR (1975) "Mini-mental state". A practical method for grading the cognitive state of patients for the clinician. J Psychiatr Res 12(3):189-198

19. Mattis S (1988) Dementia rating scale. Professional manual, Psychological Assessment Resources, Odessa

20. Sako W et al (2008) Bilateral deep brain stimulation of the globus pallidus internus in tardive dystonia. Mov Disord 23(13):1929-1931

21. Trottenberg $\mathrm{T}$ et al (2005) Treatment of severe tardive dystonia with pallidal deep brain stimulation. Neurology 64(2):344-346

22. Chang EF et al (2010) Long-term benefit sustained after bilateral pallidal deep brain stimulation in patients with refractory tardive dystonia. Stereotact Funct Neurosurg 88(5):304-310

23. Franzini A et al (2005) Long-term high-frequency bilateral pallidal stimulation for neuroleptic-induced tardive dystonia. Report of two cases. J Neurosurg 102(4):721-725

24. Shaikh AG et al (2015) Temporal profile of improvement of tardive dystonia after globus pallidus deep brain stimulation. Parkinsonism Relat Disord 21(2):116-119

25. Cheung T et al (2013) Sustained relief of generalized dystonia despite prolonged interruption of deep brain stimulation. Mov Disord 28(10): 1431-1434

26. Hebb MO et al (2007) Sustained relief of dystonia following cessation of deep brain stimulation. Mov Disord 22(13):1958-1962

27. Boulogne S et al (2014) Relapse of tardive dystonia after globus pallidus deep-brain stimulation discontinuation. J Neurol 261(8):1636-1637

28 Eltahawy HA et al (2004) Primary dystonia is more responsive than secondary dystonia to pallidal interventions: outcome after pallidotomy or pallidal deep brain stimulation. Neurosurgery 54(3):613-619 (discussion 619-21)

29. Kang UJ, Burke RE, Fahn S (1986) Natural history and treatment of tardive dystonia. Mov Disord 1(3):193-208

30. Tarsy D, Baldessarini RJ (2006) Epidemiology of tardive dyskinesia: is risk declining with modern antipsychotics? Mov Disord 21(5):589-598

31. Zutshi D, Cloud LJ, Factor SA (2014) Tardive syndromes are rarely reversible after discontinuing dopamine receptor blocking agents: experience from a University-based Movement Disorder Clinic. Tremor Other Hyperkinet Mov (N Y) 4:266 